\title{
Disigning A Novel Architecture to Reduce Stand by and Dynamic Power Dissipation for Sleepy Keeper Cmos Logic Circuits
}

\author{
Vidyavati Mallaraddi ${ }^{1}$, H.P.Rajani ${ }^{2}$ and S.S Kamate ${ }^{3}$ \\ ${ }^{1}$ Department of Electronics and Communication Engineering, KLE Dr. M.S. \\ Sheshgiri College of Engineering and Technology, Belagavi,Karnataka, India \\ ${ }^{2}$ Department of Electronics and Communication Engineering, KLE Dr. M.S. \\ Sheshgiri College of Engineering and Technology, Belagavi ,Karnataka,India \\ ${ }^{3}$ Department of Electronics \& Communication Engineering, Hirasugar \\ Institute of Technology, Nidasoshi,Karnataka,India
}

\begin{abstract}
Scaling down of transistors will directly affects the decreasing in threshold voltage. Impact of decreasing threshold voltage is increasing in leakage current. In this paper many power gating circuits are discussed for their advantages and disadvantages, based on this literature survey a novel low power state retention technique is proposed in this paper. The proposed technique with its novel architecture reduces the leakage power (static power) and dynamic power dissipations. It also gives the state retained output. Performance of the proposed circuit is evaluated on some basic circuits in terms of their total and dynamic power dissipations. Extensive SPICE simulations were carried out and the results are compared with some existing techniques. Simulated results shows that proposed technique gives the efficient power minimization and state retained standby mode output.
\end{abstract}

KEY WORDS: FEEDBACK, HPG, LOW POWER, MTMOS, ON CURRENT.

\section{INTRODUCTION}

As the moor's law says that for every eighteen months the numbers of transistors on a silicon die are going to be double, now a day's the millions of transistors are accumulated on System on Chips (SoC).as the number of transistors doubles it also decreases the size of transistor and intern the power dissipation increases. It's not a severe problem in small circuits but as the circuit size and complexity increases, the high power dissipation diminish the battery life and also it requires the extra

\section{ARTICLE INFORMATION}

*Corresponding Author: vidya.mallaraddi@gmail.com Received 12th Oct 2020 Accepted after revision 31 st Dec 2020 Print ISSN: 0974-6455 Online ISSN: 2321-4007 CODEN: BBRCBA

Thomson Reuters ISI Web of Science Clarivate Analytics USA and Crossref Indexed Journal

\section{Clarivate
Analytics}

NAAS Journal Score 2020 (4.31)

A Society of Science and Nature Publication,

Bhopal India 2020. All rights reserved.

Online Contents Available at: http//www.bbrc.in/

Doi: http://dx.doi.org/10.21786/bbrc/13.13/19 cooling and packaging costs.

$\mathrm{P}_{\text {total }}=\mathrm{P}_{\text {dynamic }}+\mathrm{P}_{\text {shortcircuit }}+\mathrm{P}_{\text {static }}$

From the above equation shows the sources of power dissipation.(1) Pdynamic due to switching activity of the input.(2) Pshortcircuit due to momentary conduction path between supply and ground .(3) Pstatic due to leakage current. The major design metrics in the VLSI circuit design are power and delay. As the supply voltage scales down the threshold voltage also should scale down. It increase the leakage current in exponential way. This increases the static power dissipation. One more design consideration is Output current that varies directly with the leakage current. As we decrease the output current, it intern decrease the leakage current but coming to the delay, propagation delay is inversely proportional to the output current as the output current decreases it increases the propagation delay.

So always there is a tradeoff between the delay and leakage power. There are many concepts for reducing

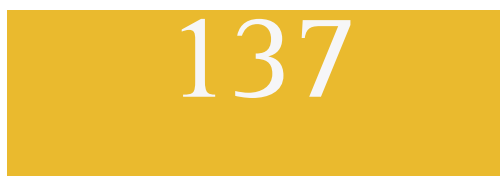


the leakage power dissipation. Those are sleep transistor, body biasing methods and leakage control transistor methods and so on. These techniques were discussed for their merits and de merits in Material and Methods section. This Section also reveals the proposed design. In Result and discussion section shows the comparative results on Power dissipations of few combinational circuits. Last Section concludes this paper.

\section{MATERIAL AND METHODS}

In (Mutoh et al., 1995) proposed the MTCMOS technique, here high threshold header and footer sleep signal operated PMOS and NMOS are inserted for basic conventional circuit. And the low threshold transistors are chosen for base logic circuit. This technique is not suitable for low power circuits. This technique takes more delay and area to produce specified output. In (Drake et al., 2003) presented the Dynamic threshold CMOS technique, in this circuit base and gate of a MOSFETS are shorted, therefore dynamically it varies the threshold voltage by the concept of forward and reverse body biasing. The main drawback of this circuit is according to (Keshavarzi et al., 1999) reverse body bias leads to the steep rise in junction leakage current due to the technology scaling. In (Hanchate et al., 2004) author proposed the Lector technique.

This circuit is a self controlled one, but the drawback of this technique is output levels are not good. In (Kim et al., 2006) author put forward the sleepy keeper approach. This approach retains the state of output in standby mode. But main drawback of this circuit is increased area and wiring lengths leads to the more delay in output. In (Lakshmikanthan et al., 2006) authors recommended the new technique sleep circuit embedded CMOS circuit. This circuit avoids the draining of battery in the standby mode by producing the zero output, but the disadvantage of this technique is the concept of state retention is not maintained. In (Katrue et al., 2008) the GALEOR technique was proposed, this technique is a self controlled design with good leakage minimization but the main drawback is output signal levels are not good. Drain gating concept was presented in (Chun et al., 2010).

In this circuit power is gated at the drain side instead of at the source side. With this concept it remarkably reduces the dynamic power dissipation but not the static power dissipation. In (Chowdhury et al., 2012) authors projected a new variable body biasing technique. In this circuit the sleep transistor threshold voltage is increased from body biasing technique so, in turn it decreases the sub threshold leakage current. But main drawback is complexity and area gets increased from this extra body biasing circuit. A GLBB technique was recommended in (Corsonello et al., 2014). This circuit gives a good delay minimization, but at cost of increased area. In (Sharma et al., 2015) it was proposed the new technique called ONOFIC. In this circuit delay and power will be get reduced from a feedback PMOS transistor.
Appropriate selection of input signal can give a better leakage minimization, but selection of input signal is needed to be done by algorithms which take extensive computational time. New LCNT approach was found by (Lorenzo et al., 2017). this circuit reduces the leakage power with a series connected two NMOS transistors in between output and pull down circuit. With the cost of less quality signal levels. In (Johannah et al., 2017) authors recommended the hybrid power gating technique, as discussed in further section it decreases the leakage power dissipation through sleep transistor stacking effect and through PMOS feedback transistor and reverse body bias technique is used to further reduce the leakage power dissipation. This feedback PMOS transistor feeds back the leakage current to input. This will reduce the dynamic power dissipation.

Proposing A New Low Power Technique: To achieve Low power dissipation in CMOS circuits, they will be incorporated by few leakage control transistors.

MTCMOS: Let us take a conventional inverter circuit. For this circuit M1 PMOS is connected between power supply and pull up circuit. This circuit controlled from V2 (sleep signal) voltage source. The M4 NMOS is connected between pull down and ground. The M4 NMOS is controlled from V3 (complement of sleep signal) voltage.

\section{Figure 1: MTCMOS circuit}

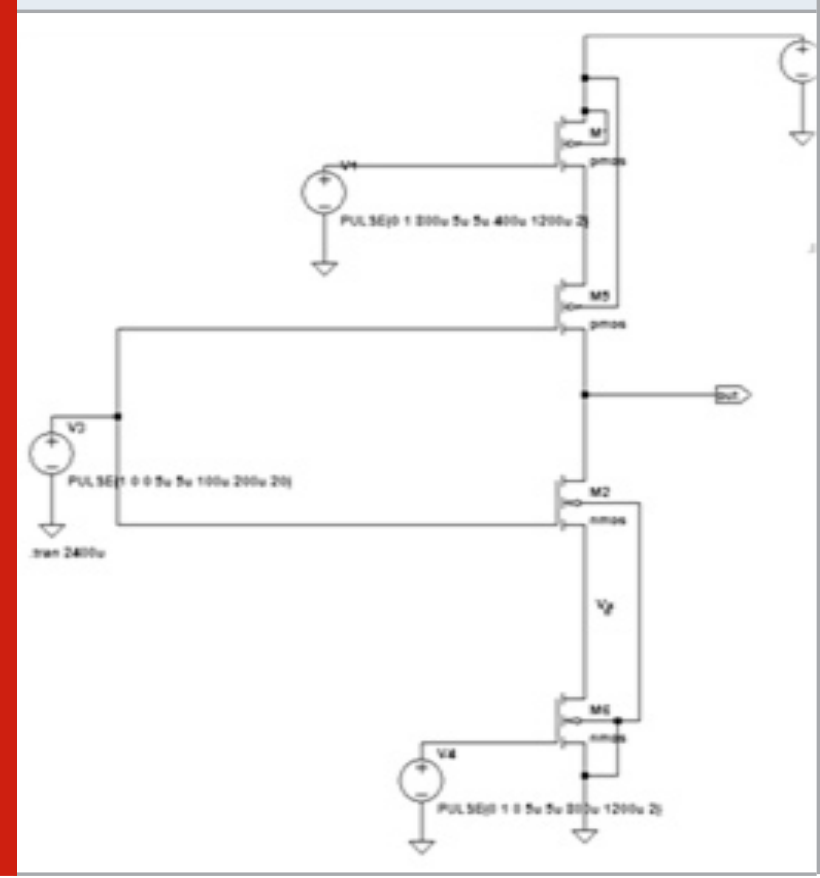

The Multi Threshold CMOS circuit will reduce the static power dissipation by reducing leakage power in sub threshold region. Because Pull down is not directly connected to the ground but through the virtual ground. It has the node potential Vx greater than ground potential. 


\section{Calculation of $\mathrm{Vx}$}

Figure 2: Analysing stack effect

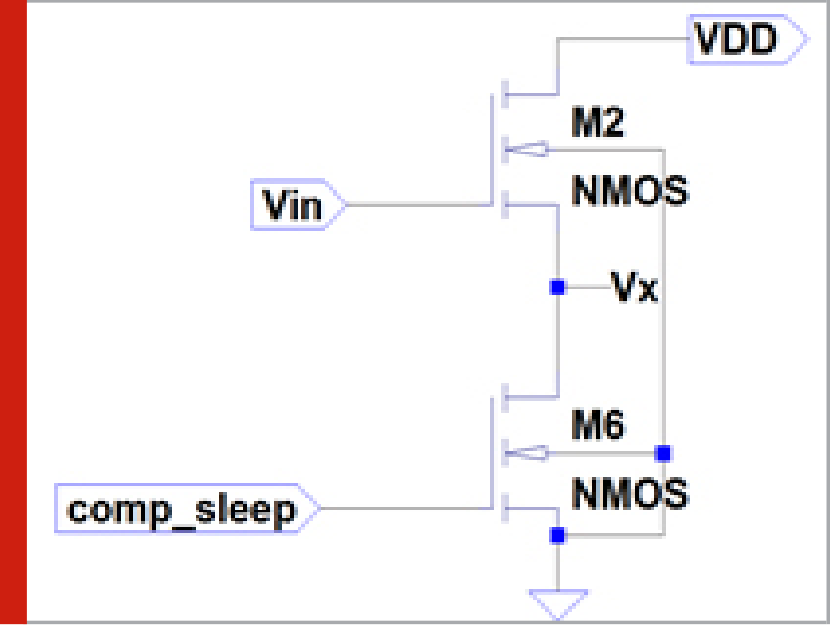

In sleep mode sleep signal is high. Comp_sleep is ' 0 ' and when Vin='0'. From Low power methodology manual: for system-on-chip design and Scaling of stack effect and its application for leakage reduction proposed by (Flynn et al., 2007; Narendra et al., 2001) Modified the standard leakage power equation by replacing ex component by 10x component.

$$
\mathrm{I}_{\text {leakage }}=\mathrm{I}_{\text {off }} 10 \frac{V g s-V t h o-K_{\gamma} V s b+\eta V d s}{\mathrm{~s}}
$$

Where I off is the sub threshold leakage current at $\mathrm{V}_{\mathrm{gs}}=0$, assume $\mathrm{V}_{\text {tho }}=0$ threshold voltage at zero bias condition and $\mathrm{V}_{\mathrm{ds}}^{\text {tho }}=\mathrm{Vdd}, \mathrm{S}$ is sub threshold slope, $\eta$ is DIBL coefficient $\mathrm{K}_{\gamma}$ is body effect coefficient. From stack effect analyzing circuit (Fig.2). Leakage current of M2 and M6 transistors are given by

$$
\begin{aligned}
& \mathbf{I}_{\text {leakM1 }}=\mathrm{I}_{\text {off }} 10 \frac{-V x-K, V x+\eta(V d d-V x)}{\mathrm{s}} \\
& \mathrm{I}_{\text {leakM6 }}=\mathrm{I}_{\text {off }} \quad 10^{\frac{\eta V x}{\mathrm{~s}}}
\end{aligned}
$$

Vx is obtained by equating $\mathrm{I}_{\text {leak }} \mathrm{M} 1$ and $\mathrm{I}_{\text {leak }} \mathrm{M} 6$ because M1 and M6 are two series connected MOSFETS, so same current is flows in both the transistors.

Therefore,

$$
-V x-K_{\gamma} V x+\eta(V d d-V x)=\eta V x
$$

Vx can be obtained as,

$$
V x=\frac{\eta V d d}{1+2 \eta+K_{y}}
$$

Substituting equation (5) in (3) or (4)

$$
\mathrm{I}_{\text {stack }} \simeq \mathrm{I}_{\mathrm{off}} 10^{\frac{\eta V d d}{\mathrm{~s}}}
$$

According to (Kavitha et al., 2016) assume $s=100 \mathrm{mv} /$ decade and $\eta=100 \mathrm{mv} / \mathrm{v}$ for $65 \mathrm{~nm}$ technology. By substituting these values in Istack. It shows stacking effect will minimize the leakage current by a factor of nearly 10.It shows decrease in leakage current will reduce the leakage power dissipation in turn static power dissipation.

Novel Hybrid Strucure: In (Johannah et al., 2017) authors recommended architecture i.e. novel hybrid structure.

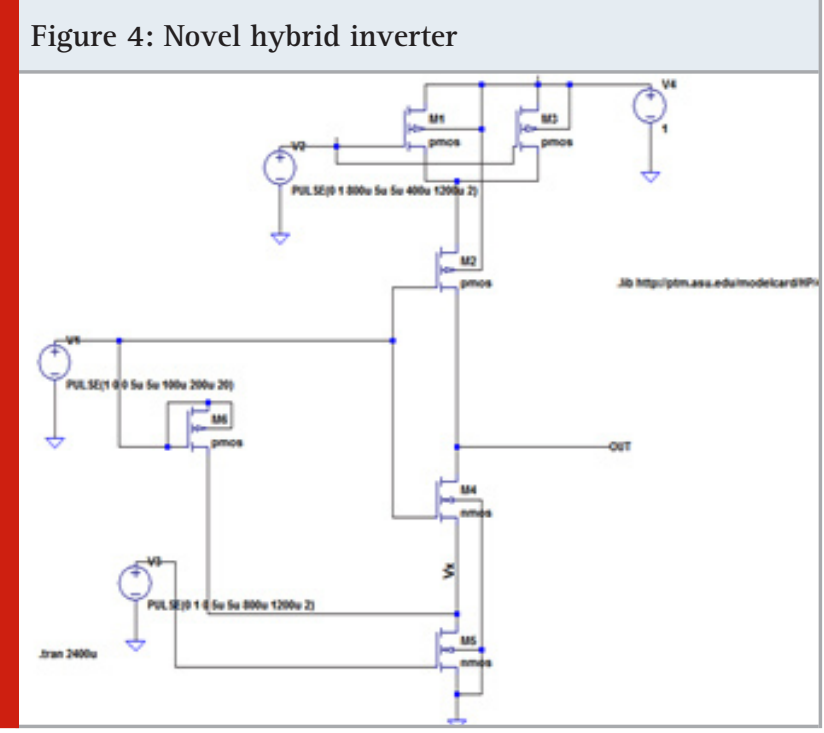

On the basic MTCMOS circuit, this Novel Hybrid structure is evolved. The extra PMOS M6 is added in the path from Vx to V1 (input voltage).V1 acts as drain and gate of M6.In active mode, when V1 (input) ='0' all PMOS transistors M1, M2, M3 and M6 are conducting and NMOS M4 is in OFF state. This combinations of inputs gives Vout=VDD. When N1 is in OFF state it tries to pull down the Vout through OFF state leakage current. Even this current is small due to stack effect which discussed previously; this little leakage current will flow from Vx to V1. This reduces the ON current of PMOS M3 and thereby reducing the dynamic power dissipation to maintain this PMOS M3 in ON state, in this way the novel Hybrid structure reduces the leakage and dynamic power dissipation.

Figure 4a: Proposed technique

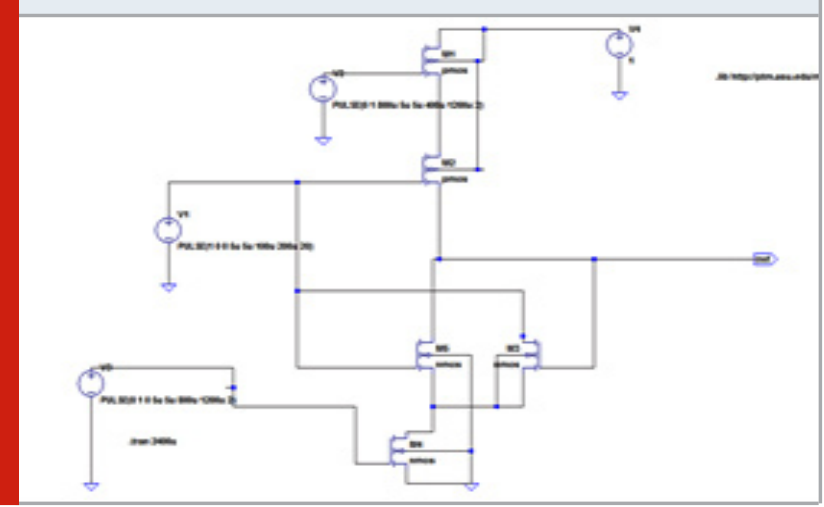

Proposed Technique: The proposed technique combines the MTCMOS and Novel Hybrid structure to reduce the 
leakage as well the dynamic power dissipation as shown below.

The proposed technique is also evolved from the MTCMOS architecture. Advantages of MTCMOS architecture are 1) It incorporates the sleep transistors for standby mode of operation.

2) This architecture retains its state when it comes out from standby mode.

3) From these sleep transistors leakage power dissipation reduces effectively.

In Novel Hybrid structure the dynamic power dissipation is reduced through feedback PMOS transistor. This feedback PMOS is connected between virtual ground and ground node to input voltage, to feed back the leakage current to input. In similar way the proposed technique [Fig.3] feedback path from Vx to V1(input) is constructed from NMOS M3 .whenever the input is ' 0 ' the output becomes 'Vdd' this Vout is gate voltage for the for the proposed design feedback NMOS M3 transistor. Due to Vout=Vdd the NMOS M3 gets ON. Whatever the leakage current flowing in $\mathrm{Vx}$ node is fed back to input through this NMOS M3 transistor, like this way it increases the ON current of Pull Up PMOS M2.both Proposed and Novel Hybrid inverter structure are used to decrease the static power dissipation through stack effect, to retain the output state after sleep mode using header and footer sleep transistors and to reduce the dynamic power dissipation through feedback transistors.

Figure 5: Proposed NAND gate

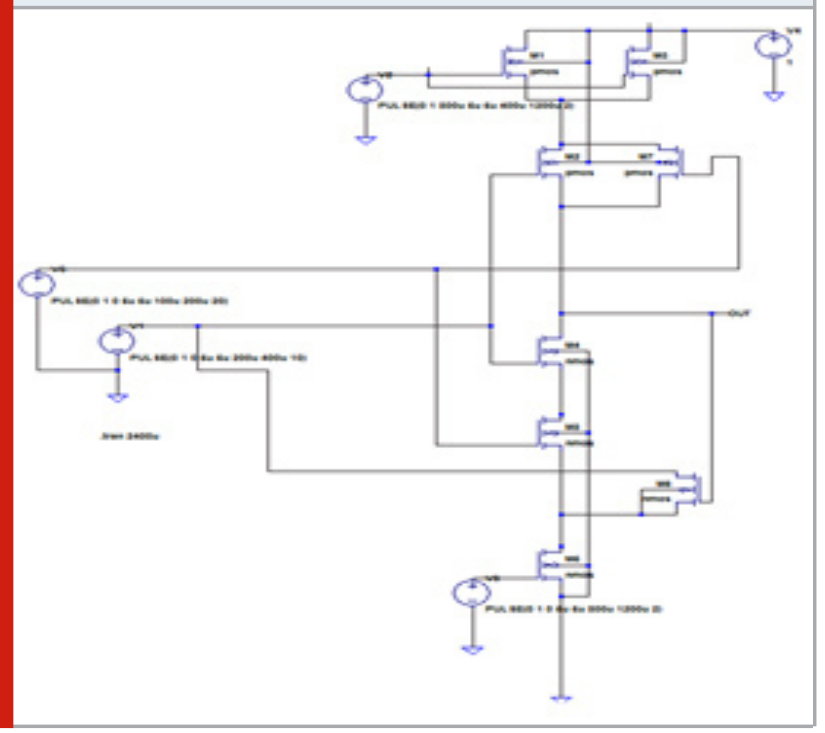

Advantages of proposed technique over Novel Hybrid structure.

1. The proposed design will efficiently reduce the total power dissipation by reducing the dynamic power dissipation. This is analyzed by PMOS versus NMOS as feedback transistors. In (Shuler 2017) it was strongly recommended that PMOS transistors, due to the use of holes rather than electrons as carriers are slower and carry less current. As a result of the latter they must be two to three times larger to balance with paired NMOS transistors. For radiation events, the PMOS transistors are simply larger and slower and contain more charges and are therefore harder to upset. So, the larger PMOS transistor is inefficient compared to NMOS transistor.

2. Another disadvantage of Novel Hybrid structure is using of reverse body biasing in feedback PMOS to reduce further leakage power. It's known that using the reverse body bias will increase the stress on the device. Over the time it decreases the performance as well the life time of a device. One of drawback it is observed from the reverse body biasing is decreasing of break down voltage of a device. But proposed architecture is free from reverse body biasing effects.

Figure 6: Proposed One bit full adder

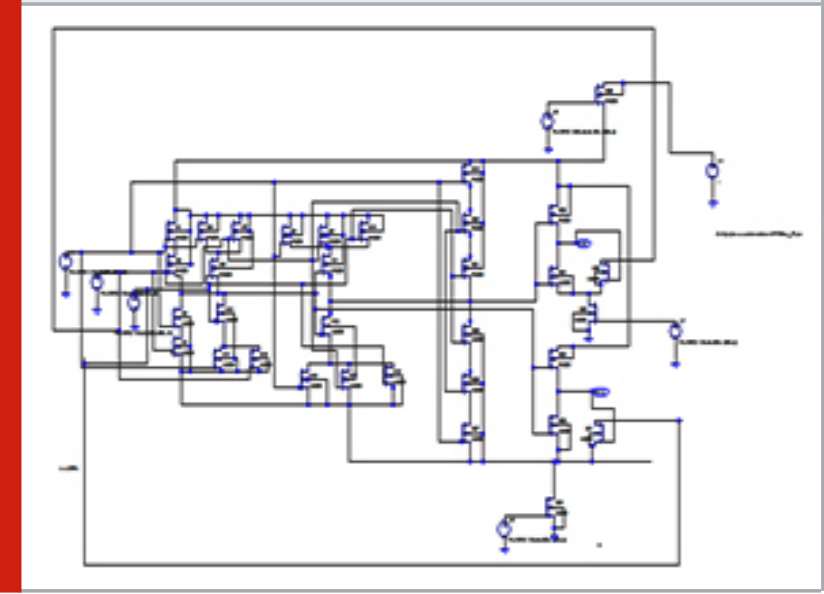

In the proposed NAND gate the virtual ground is connected to the source of the feedback transistor and drain is connected to any one of the input.

Waveform 1. Proposed inverter logic circuit output with input, total power and dynamic power dissipations.

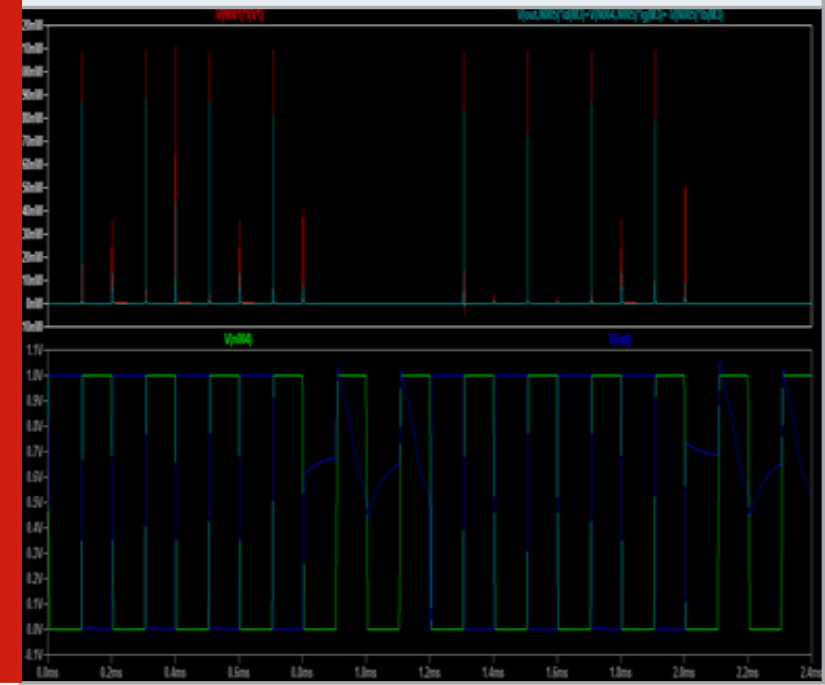

In the proposed one bit full adder circuit leakage current at the SUM output is fed back to the V2 input and leakage current at the carry output is fed back to the one more 
input V3.using this leakage current feed backing concept it noticeably reduces the total power dissipation.In the proposed one bit full adder circuit leakage current at the SUM output is fed back to the V2 input and leakage current at the carry output is fed back to the one more input V3.using this leakage current feed backing concept it noticeably reduces the total power dissipation.

Table 1. Comparison of power dissipation in Inverter circuit using different architectures

\begin{tabular}{|l|c|c|c|}
\hline sl.no & $\begin{array}{c}\text { Design } \\
\text { architecture }\end{array}$ & $\begin{array}{c}\text { Average total } \\
\text { power dissipation } \\
\text { (over 2.4ms) }\end{array}$ & $\begin{array}{c}\text { Average dynamic } \\
\text { power } \\
\text { dissipation }\end{array}$ \\
\hline 1 & Conventional & $608.8 \mathrm{pW}$ & $333.8 \mathrm{pW}$ \\
\hline 2 & MTCMOS & $397.44 \mathrm{pW}$ & $185.98 \mathrm{pW}$ \\
\hline 3 & Sleep circuit Embedded & $496.6 \mathrm{pW}$ & $247.68 \mathrm{pW}$ \\
\hline 4 & CMOS circuit & $439.6 \mathrm{pW}$ & $86.3 \mathrm{pW}$ \\
\hline 6 & Drain gating & $376.65 \mathrm{pW}$ & $185.98 \mathrm{pW}$ \\
\hline 7 & Novel Hybrid Gating & $373 \mathrm{pW}$ & $197.96 \mathrm{pW}$ \\
\hline 8 & Proposed Design & $362 \mathrm{pW}$ & $75.74 \mathrm{pW}$ \\
\hline
\end{tabular}

Table 2. Effect of technology scaling on total and dynamic power dissipation in proposed technique

\begin{tabular}{|c|c|c|c|c|}
\hline sl.no & $\begin{array}{c}\text { Different } \\
\text { Technology } \\
\text { nodes }\end{array}$ & $\begin{array}{c}\text { Scaled } \\
\text { downed } \\
\text { Supply voltage }\end{array}$ & $\begin{array}{c}\text { Average Total } \\
\text { power dissipation } \\
\text { (over 2.4ms) }\end{array}$ & $\begin{array}{c}\text { Average Dynamic } \\
\text { power } \\
\text { dissipation }\end{array}$ \\
\hline 1 & $45 \mathrm{~nm}$ & $1.1 \mathrm{~V}$ & $853.79 \mathrm{pW}$ & $198.27 \mathrm{pW}$ \\
\hline 2 & $32 \mathrm{~nm}$ & $1 \mathrm{~V}$ & $297.52 \mathrm{pW}$ & $57.29 \mathrm{pW}$ \\
\hline 3 & $22 \mathrm{~nm}$ & $0.9 \mathrm{~V}$ & $52.3 \mathrm{pW}$ & $11.47 \mathrm{pW}$ \\
\hline 4 & $16 \mathrm{~nm}$ & 0.8 & $26.5 \mathrm{pW}$ & $6.2 \mathrm{pW}$ \\
\hline
\end{tabular}

Waveform 2. Proposed NAND logic circuit output with input, total power and dynamic power dissipations.

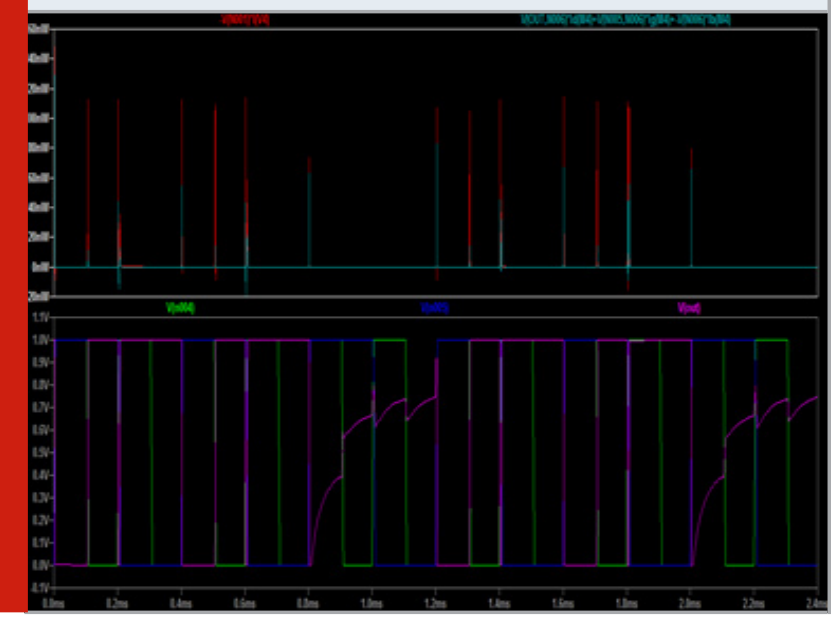

\section{RESULTS AND DISCUSSION}

The inverter logic circuit is implemented using $45 \mathrm{~nm}$ technology (taking from Low Power PTM library files) and by applying the $1 \mathrm{~V}$ power supply in LTspiceXVII.
From the Table 1, it proves that the total as well dynamic power dissipation will be considerably reduced as compared to other architectures.

From the above comparison Table 2, it is observed that, with technology scaling proposed low power architecture works well to reduce the total as well dynamic power dissipation remarkably.

Waveform 3. Resultant waveform of the proposed One bit Full Adder

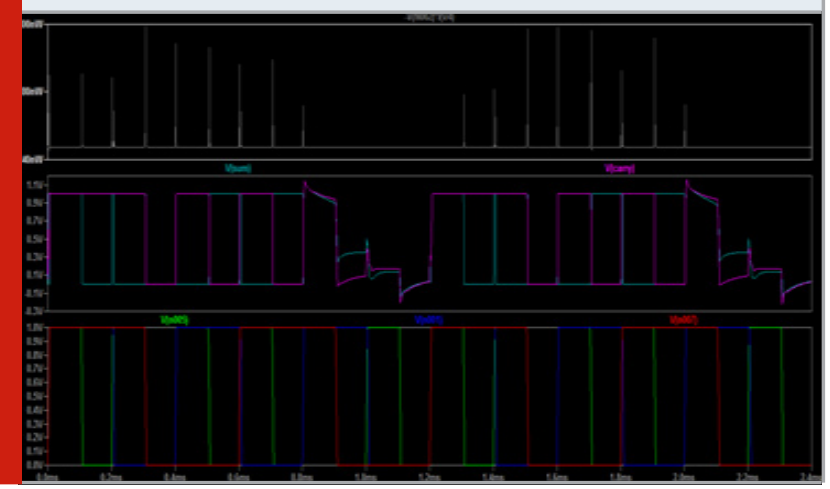


Table 3. Comparison of average total power and dynamic Power dissipation in NAND logic circuit constructed from Different design architectures.

\begin{tabular}{|c|c|c|c|}
\hline sl.no & $\begin{array}{c}\text { Design } \\
\text { Architecture }\end{array}$ & $\begin{array}{c}\text { Average Total } \\
\text { power dissipation } \\
\text { (over 2.4ms) }\end{array}$ & $\begin{array}{c}\text { Average Dynamic } \\
\text { power dissipation }\end{array}$ \\
\hline 1 & Conventional & $648.46 \mathrm{pW}$ & $291.25 \mathrm{pW}$ \\
\hline 2 & MTCMOS & $438.22 \mathrm{pW}$ & $173.99 \mathrm{pW}$ \\
\hline 3 & Novel Hybrid Gating & $433.01 \mathrm{pW}$ & $139.62 \mathrm{pW}$ \\
\hline 4 & Proposed NAND gate & $288.74 \mathrm{pW}$ & $29.9 \mathrm{pW}$ \\
\hline
\end{tabular}

Table 4. Comparison of average total power dissipation in Designing One bit full adder using low power $45 \mathrm{~nm}$ technology.

\begin{tabular}{|l|c|c|}
\hline sl.no & $\begin{array}{c}\text { Design } \\
\text { Architecture }\end{array}$ & $\begin{array}{c}\text { Average Total } \\
\text { power dissipation } \\
\text { (over 2.4ms) }\end{array}$ \\
\hline 1 & $\begin{array}{c}\text { Conventional one } \\
\text { bit full adder }\end{array}$ & $2.371 \mathrm{nW}$ \\
\hline 2 & $\begin{array}{c}\text { Proposed one bit } \\
\text { full adder }\end{array}$ \\
\hline
\end{tabular}

\section{CONCLUSION}

The aim of VLSI circuit design is to miniature the electronic circuits and in turn to reduce the power dissipation. As the technology scales down it decreases the threshold voltage which leads to the increase in leakage power. In this paper, through literature survey many leakage power techniques are analyzed to find there advantages and disadvantages. These techniques are compared with proposed power reduction technique. We found the proposed technique gives relatively good dynamic and total power reduction. In future we can implement this technique to design a low power processor.

\section{REFERENCES}

Corsonello, P., Lanuzza, M. and Perri, S., 2014. Gate-level body biasing technique for high-speed sub-threshold CMOS logic gates. International journal of circuit theory and applications, 42(1), pp.65-70.

Chun, J.W. and Chen, C.R., 2010, November. A novel leakage power reduction technique for CMOS circuit design. In 2010 International SoC Design Conference (pp. 119-122). IEEE.

Chowdhury, M.A.J., Rizwan, M.S. and Islam, M.S., 2012. An efficient VLSI design approach to reduce static power using variable body biasing. World Academy of science, Engineering and Technology, 64(2), pp.7-15.

Drake, A.J., Zamdmer, N., Nowka, K.J. and Brown, R.B.,
2003, September. Analysis of the impact of gate-body signal phase on DTMOS inverters in 0.13 um PD-SOI. In IEEE international SOI conference (pp. 99-100). (2003).

Flynn, D., Aitken, R., Gibbons, A. and Shi, K., 2007. Low power methodology manual: for system-on-chip design. Springer Science \& Business Media.

Hanchate, N. and Ranganathan, N., 2004. LECTOR: a technique for leakage reduction in CMOS circuits. IEEE Transactions on Very Large Scale Integration (VLSI) Systems, 12(2), pp.196-205.

Johannah, J.J., Korah, R. and Kalavathy, M., 2017. Standby and dynamic power minimization using enhanced hybrid power gating structure for deepsubmicron CMOS VLSI. Microelectronics Journal, 62, pp.137-145.

Katrue, S. and Kudithipudi, D., 2008, August. GALEOR: Leakage reduction for CMOS circuits. In 2008 15th IEEE International Conference on Electronics, Circuits and Systems (pp. 574-577). IEEE.

Kavitha, M. and Govindaraj, T., 2016. Low-power multimodal switch for leakage reduction and stability improvement in SRAM cell. Arabian Journal for Science and Engineering, 41(8), pp.2945-2955.

Kim, S.H. and Mooney, V.J., 2006, October. Sleepy keeper: a new approach to low-leakage power VLSI design. In 2006 IFIP International Conference on Very Large Scale Integration (pp. 367-372). IEEE.

Keshavarzi, A., Narendra, S., Borkar, S., Hawkins, C., Roy, K. and De, V., 1999, August. Technology scaling behavior of optimum reverse body bias for standby leakage power reduction in CMOS IC's. In Proceedings of the 1999 international symposium on Low power electronics and design (pp. 252-254).

Lakshmikanthan, P. and Nuñez, A., 2006, September. A novel methodology to reduce leakage power in cmos complementary circuits. In International Workshop on Power and Timing Modeling, Optimization and Simulation (pp. 614-623). Springer, Berlin, Heidelberg. 
Lorenzo, R. and Chaudhury, S., 2017. LCNT-an approach to minimize leakage power in CMOS integrated circuits. Microsystem Technologies, 23(9), pp.4245-4253.

Mutoh, S.I., Douseki, T., Matsuya, Y., Aoki, T., Shigematsu, S. and Yamada, J., 1995. 1-V power supply high-speed digital circuit technology with multithreshold-voltage CMOS. IEEE Journal of Solidstate circuits, 30(8), pp.847-854.

Narendra, S., De, V., Antoniadis, D., Chandrakasan, A. and Borkar, S., 2001, August. Scaling of stack effect and its application for leakage reduction. In Proceedings of the 2001 international symposium on Low power electronics and design (pp. 195-200).

Sharma, V.K., Pattanaik, M. and Raj, B., 2015. INDEP approach for leakage reduction in nanoscale CMOS circuits. International Journal of Electronics, 102(2), pp.200-215.

Shuler, R.L., 2017, July. Configuration Tests of RHBD Library with DICE, TAG4, dual rail, TMR and New 10T Voting Latch using UMC 65 and XFAB 180 nm. In 2017 IEEE Radiation Effects Data Workshop (REDW) (pp. 1-6). IEEE. 